\title{
Gastrointestinal toxicities of immune checkpoint inhibitors: a multicenter retrospective analysis
}

\author{
Christine Shieh ${ }^{a}$, Divya Chalikonda ${ }^{b}$, Peter Block ${ }^{a}$, Brianna Shinn ${ }^{b}$, C. Andrew Kistler ${ }^{b}$ \\ Thomas Jefferson University Hospital, USA
}

\begin{abstract}
Background Immune checkpoint inhibitors are monoclonal antibodies that augment immune cell function and are used to treat malignancy. However, they may cause proinflammatory adverse events. This study investigated gastrointestinal (GI) adverse events associated with specific immune checkpoint inhibitors.

Methods Charts of patients aged $>18$ years with a solid tumor who underwent treatment with immune checkpoint inhibitors between 1st April 2011 and 1st August 2019 were reviewed for GI toxicities. Clinical data, including interventions, treatment duration and outcomes, were recorded.

Results One hundred patients were included in the study, of whom 22 experienced a GI adverse event directly attributable to immune checkpoint inhibitors. Transaminitis $(9 / 22 ; 40.9 \%)$ and colitis $(8 / 22 ; 36.3 \%)$ were most prevalent. The majority of events occurred within 4 cycles of treatment onset and were most prevalent with the nivolumab + ipilimumab combination (7/12; $58.3 \%)$. In $91 \%$ of cases (20/22), patients showed improvement or resolution of the event. Among the colitis cases, there was a significant difference $(\mathrm{P}=0.004)$ in recovery time between those who received infliximab and those who did not. Despite symptom resolution, only 7/22 were left on the same or part of the same treatment regimen.

Conclusions Most patients experienced their GI adverse events within 4 cycles of starting treatment, the most common being transaminitis and colitis. Nivolumab + ipilimumab dual therapy was most strongly associated with colitis. Most adverse events self-resolved, with infliximab being particularly helpful in improving colitis symptoms. However, most patients were unable to tolerate the same immunotherapy regimen and ultimately expired.
\end{abstract}

Keywords Oncology, checkpoint inhibitor, gastrointestinal, colitis

Ann Gastroenterol 2020; 33 (6): 1-7

\section{Introduction}

Immune checkpoint inhibitors (ICIs) make up a novel class of drug that has revolutionized the therapeutic approach to various malignancies and has consequently become increasingly common in daily oncology practice. These medications facilitate the inhibition of down-regulators of the immune system, thereby augmenting immune cell function via various pathways

Department of a Medicine (Christine Shieh, Peter Block); ${ }^{b}$ Division of Gastroenterology and Hepatology (Divya Chalikonda, Brianna Shinn, C. Andrew Kistler), Thomas Jefferson University Hospital, USA

Conflict of Interest: None

Correspondence to: C. Andrew Kistler, MD, PharmD, 132 South $10^{\text {th }}$ Street, Suite 480 Main Building, Philadelphia, PA 19107, USA, e-mail: charles.kistler@jefferson.edu

Received 2 May 2020; accepted 14 July 2020; published online 12 October 2020

DOI: https://doi.org/10.20524/aog.2020.0552 and eventually resulting in increased activation of T-cells [1,2]. Several monoclonal antibodies have been approved by the Food and Drug Administration for use in various malignancies; these include programmed cell death protein ligand inhibitors (PD-L1 inhibitors: atezolizumab, avelumab, durvalumab), programmed cell death protein inhibitors (PD-1 inhibitors: nivolumab, pembrolizumab), and cytotoxic T-lymphocyte associated antigen inhibitors (CTLA-4 inhibitors: ipilimumab, tremelimumab) [3].

By augmenting the activity of the immune system, ICIs can induce proinflammatory adverse events, termed immunerelated adverse events (irAEs) [2,4]. Though irAEs can affect any organ, the endocrine glands, skin, gastrointestinal (GI) tract, and liver are most frequently implicated [2-4]. GI tract involvement is one of the most common types of irAEs, constituting about $30-50 \%$ of the total $[5,6]$. The exact pathophysiology of irAEs is not completely understood, but is thought to be a manifestation of a disruption of immune system homeostasis, resulting in altered T-cell and cytokine responses $[4,5]$. Studies thus far have shown that the organ 
systems affected by ICIs vary based on the specific molecule targeted, whether PD-1, PD-L1 or CTLA-4 [2,5]. However, given their relatively recent increased use, the frequency with which various ICIs cause organ-specific AEs, particularly within the GI tract, has yet to be fully elucidated.

Beyond frequency, several aspects of irAEs remain unclear, including approach to treatment, treatment outcome and timeframe of presentation and resolution. In turn, the identification and management of irAEs have significant heterogeneity in practice, while a significant amount of research and data is still accumulating. GI and liver AEs frequently result in referrals to gastroenterologists and hepatologists. Typical GI irAEs noted include immune-mediated colitis (8-22\%), hepatotoxicity (4-11\%), pancreatitis (10-15\%), and non-specific symptoms of nausea, vomiting and diarrhea (27-54\%) [5-7]. Early recognition and suspicion for irAEs are critical to prevent detrimental clinical outcomes such as liver failure, toxic megacolon or bowel perforation. Therefore, we aimed to identify the GI irAEs associated with ICIs in this multi-center study and to describe the prevalence, management, and clinical outcomes of GI irAEs to further fill the current research void regarding these agents.

\section{Patients and methods}

\section{Patients}

The electronic health record (EHR) for Thomas Jefferson University Hospital and Methodist Hospital was queried to identify patients aged over 18 years with a solid tumor cancer who underwent treatment with ICIs between 1st April 2011 and 1st August 2019. Demographic data recorded included type and stage of cancer, Eastern Cooperative Oncology Group (ECOG) Performance Status, and smoking and alcohol use. Patients were not receiving concomitant active chemotherapy. Our protocol was approved by the institutional review board. Patient charts were screened for ICD-10 codes with the following GI AEs: colitis, hepatitis, pancreatitis, elevated bilirubin or transaminases, nausea, vomiting, diarrhea, and abdominal pain. These charts were reviewed for GI AEs attributed to the ICIs according to clinical documentation by the oncologist. Attributing AEs to immunotherapy was based on the patient's symptoms, improvement upon discontinuation of the immunotherapy and/or treatment with steroids. Clinical data for each GI AE recorded included grading by the Common Terminology Criteria for Adverse Events scale, number of cycles prior to the GI AE, interventions, recovery time, and clinical outcomes.

\section{Statistical analysis}

Categorical and continuous variables were summarized using proportions and percentages. Mean \pm standard deviation was calculated for continuous variables when possible. Univariate analysis was performed on recovery time.

\section{Results}

A total of 100 patients met the inclusion criteria and were screened for GI AEs while on ICIs. Patient characteristics and immunotherapy treatment are presented in Table 1 . Seventyfive patients were functional, with ECOG scores of 0 or 1 , while 85/100 had advanced stage cancer, either Stage III or IV. The most common cancers represented in our study were melanoma (41/100) and lung cancer (34/100).

Of the 100 patients, 22 experienced a GI AE closely attributed to the use of ICIs. These GI AEs were distributed as follows: 9/22 patients with transaminitis, $8 / 22$ with colitis, $3 / 22$ with unspecified nausea and diarrhea, and $2 / 22$ with pancreatitis (Table 2). Patients received an average of 4 cycles of immunotherapy prior to experiencing side-effects. For the 2 most common side-effects seen, transaminitis was associated with nivolumab use in 6/9 cases and colitis was associated with the dual regimen of nivolumab/ ipilimumab in 5/8 cases (Fig. 1). In $91 \%$ of cases (20/22), patients had improvement or resolution of the GI AE. There was no significant difference in recovery time between transaminitis and colitis $(\mathrm{P}=0.38)$; the mean recovery time was $43 \pm 36$ days. However, among the colitis cases, there was a significant difference $(\mathrm{P}=0.004)$ in recovery time between those who received infliximab (IFX) (10.75 \pm 11.3 days) and those who did not ( $63.75 \pm 16.3$ days). Despite resolution of the GI symptoms, only 7/22 were maintained on the same or part of the same treatment regimen. Ultimately, the mortality rate for this group of patients experiencing GI AE on immunotherapy was $41 \%(9 / 22)$, although the GI AE were not necessarily the cause of death. Of the deaths, only one involved
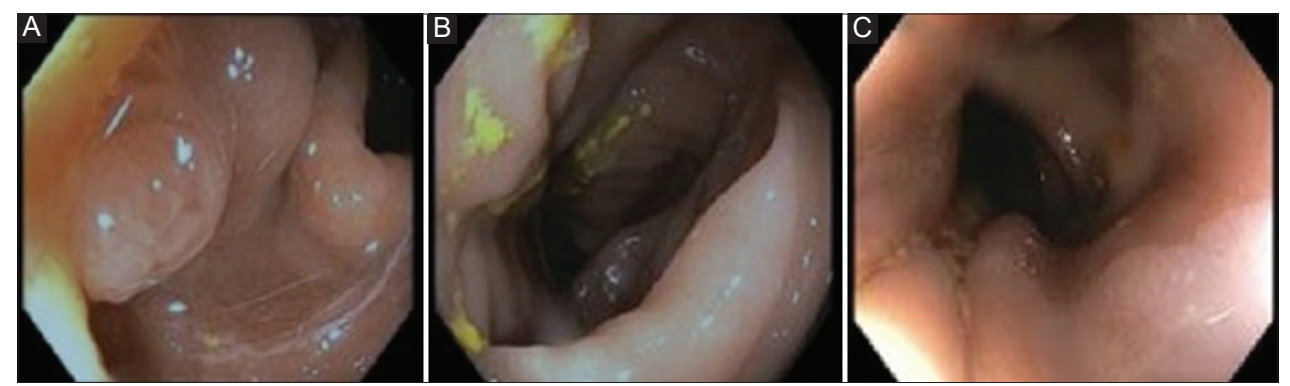

Figure 1 Immunotherapy induced colitis on endoscopy. This patient had symptoms consistent with Grade 2 colitis on the Common Terminology Criteria for Adverse Events scale. The patient's endoscopy showed (A) mild congestion in the proximal small bowel and (B, C) diffuse congestion throughout the colon. Biopsies were positive for active colitis with intraepithelial lymphocytosis highly suggestive of pembrolizumab toxicity. The patient received one dose of infliximab and showed immediate improvement in symptoms as well as a prednisone taper 
Table 1 Patient characteristics

\begin{tabular}{|c|c|}
\hline Parameter & Value \\
\hline \multicolumn{2}{|l|}{ Age $( \pm S D)$} \\
\hline Male & $66.0 \pm 12.4$ \\
\hline Female & $67.9 \pm 10.2$ \\
\hline \multicolumn{2}{|l|}{ Sex } \\
\hline Male & 60 \\
\hline Female & 40 \\
\hline Type of cancer & $\#$ of cases (of 100) \\
\hline \multicolumn{2}{|l|}{ Melanoma } \\
\hline Eye & 7 \\
\hline Skin, BRAF mut & 6 \\
\hline Skin, BRAF wt & 18 \\
\hline Skin, BRAF unknown & 10 \\
\hline \multicolumn{2}{|l|}{ Lung } \\
\hline Squamous cell & 10 \\
\hline Adenocarcinoma & 21 \\
\hline Small cell & 2 \\
\hline Large cell & 1 \\
\hline GI cancer & 6 \\
\hline Head and neck & 5 \\
\hline Prostate & 3 \\
\hline Renal & 4 \\
\hline Breast & 2 \\
\hline Hodgkin's & 2 \\
\hline Uterine & 1 \\
\hline Vulvar & 1 \\
\hline Bladder & 1 \\
\hline \multicolumn{2}{|l|}{ ECOG } \\
\hline 0 & 36 \\
\hline 1 & 39 \\
\hline 2 & 9 \\
\hline 3 & 5 \\
\hline 4 & 1 \\
\hline \multicolumn{2}{|l|}{ Stage } \\
\hline I & 4 \\
\hline II & 9 \\
\hline III & 18 \\
\hline IV & 67 \\
\hline Unclear & 2 \\
\hline \multicolumn{2}{|l|}{ Social factors } \\
\hline Former smoker & 44 \\
\hline Active smoker & 8 \\
\hline Active alcohol use & 40 \\
\hline Underlying GI/autoimmune comorbidities & 1 collagenous colitis \\
\hline \multicolumn{2}{|l|}{ Immunotherapy regimens } \\
\hline Pembrolizumab & 42 \\
\hline Nivolumab & 33 \\
\hline Nivolumab + ipilimumab & 12 \\
\hline Nivolumab + brentuximab & 1 \\
\hline Durvalumab & 5 \\
\hline Atezolizumab & 3 \\
\hline Ipilimumab & 4 \\
\hline
\end{tabular}

a direct discharge home to hospice after being hospitalized for a GI AE. The remaining 8 deaths occurred later, after patients had recovered from the $\mathrm{GI} \mathrm{AE}$ but were unable to continue on the same immunotherapy regimen.
The distribution of side effects for each chemotherapy regimen is shown in Fig. 2. The majority of patients on nivolumab + ipilimumab (58.3\%; 7/12) experienced a GI AE. Among those on nivolumab monotherapy, 26.1\% (6/23) of patients had a GI AE, while for those on pembrolizumab monotherapy $11.9 \%(5 / 42)$ had a GI AE. The numbers of cases for the other immune checkpoint inhibitor regimens were small, so other patterns were difficult to appreciate.

\section{Discussion}

As an increasing number of patients are treated with ICIs, the incidence and relevance of GI AEs are also increasing. In our study of 100 patients, $22 \%$ had documented GI AEs. This is less than the current reported range of $30-50 \%$; the difference may be due to our limited cohort size and the constraint of relying on chart documentation for data [8]. Of the GI AEs, transaminitis (41\%) and colitis (36.3\%) were most commonly encountered in our cohort. In the past, colitis has been described as the most common GI side-effect after the administration of ICIs, while hepatitis has only been observed in $<5 \%$ of cases $[8,9]$. This discrepancy in prevalence may be attributed to the asymptomatic nature of some patients with elevated transaminases whose AEs therefore go undetected.

In general, CTLA-4 inhibitors such as ipilimumab and tremelimumab have higher rates of irAEs than PD-1/L1 inhibitors such as nivolumab and pembrolizumab, with $90 \%$ occurring in the former and $70 \%$ occurring in the latter [2,10]. Despite this, in our cohort transaminitis was most commonly seen with the PD-1/L1 inhibitor nivolumab. Colitis was most commonly experienced by patients on both nivolumab and ipilimumab. It is unclear if this observation is due to the use of ipilimumab in the dual-therapy regimen or to the additive effect of nivolumab. Diarrhea has been reported in up to $30 \%$ of patients following CTLA- 4 therapy, with $5 \%$ having severe colitis $[4,11]$. Only $<4 \%$ of diarrhea cases have been attributed to anti-PD-L1 therapy $[4,12]$. However, Bajwa et al conducted a meta-analysis review of all case reports/ case series in the PubMed database between 2016 and 2018 and found 14 cases of colitis, over half of which were attributed to nivolumab [6]. The prevalence of colitis on dual therapy regimen in our study may therefore be due to the additive side-effects of both drugs together. Soldatos et al conducted a large retrospective study of irAEs after the use of nivolumab alone, ipilimumab alone, or the 2 together to treat melanoma, and found that the combination of the 2 led to an expanded toxicological profile that included the side-effect profile of both agents, with higher incidences of colitis, rash, and pyrexia [13]. Patients on this combination regimen should therefore be observed carefully for early signs of worsening diarrhea.

Nearly all of our patients had improvement in their irAEs. The majority of transaminitis and pancreatitis cases were improved by holding immunotherapy and observing. Two of the higher-grade cases of transaminitis received steroids, while both cases of highgrade pancreatitis did not and still improved. The 8 cases of colitis received a course of prednisone. Of the 8 cases, 4 received IFX and had a notably quicker recovery rate in terms of symptomatology 


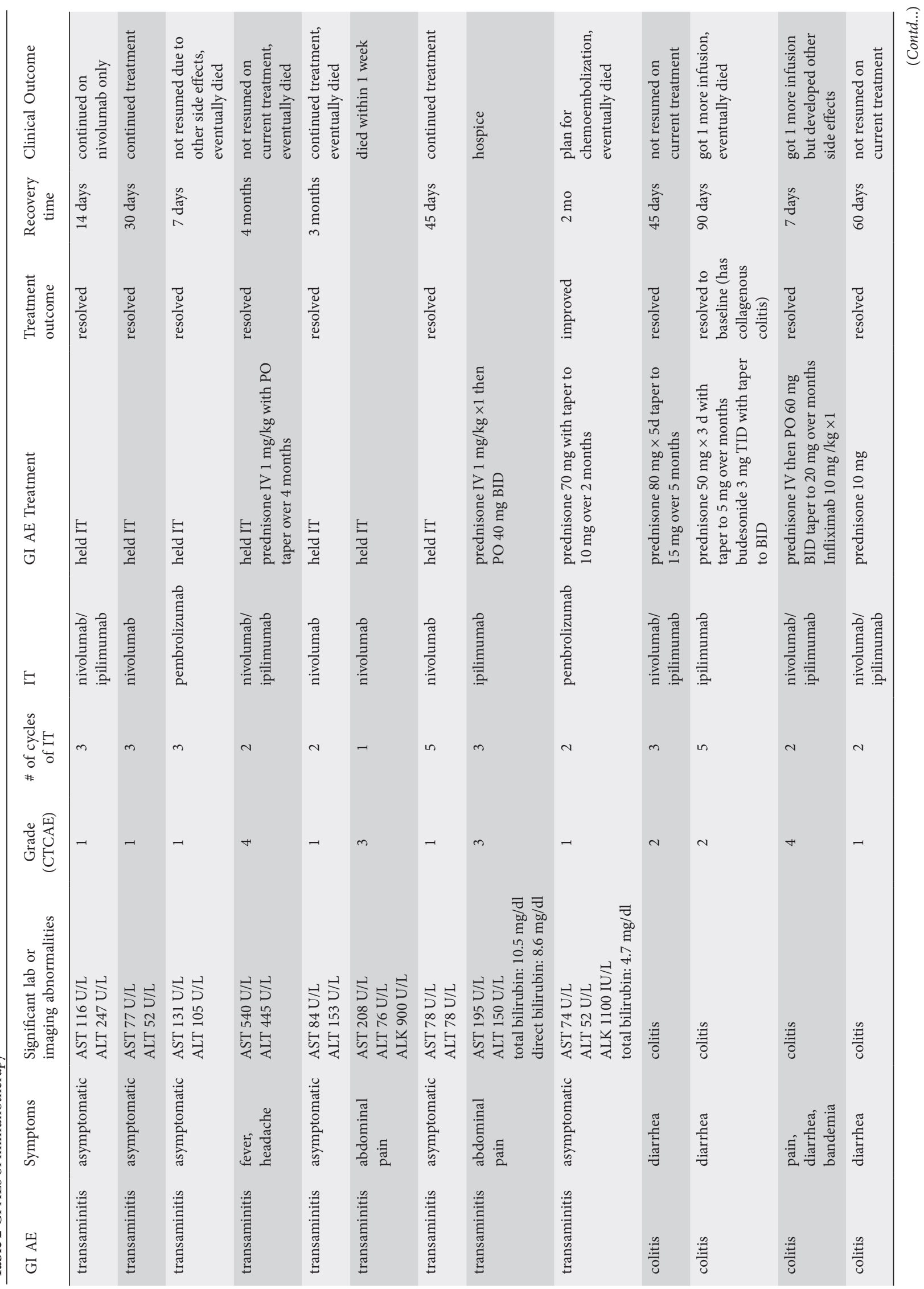


GI toxicities of immune checkpoint inhibitors 5

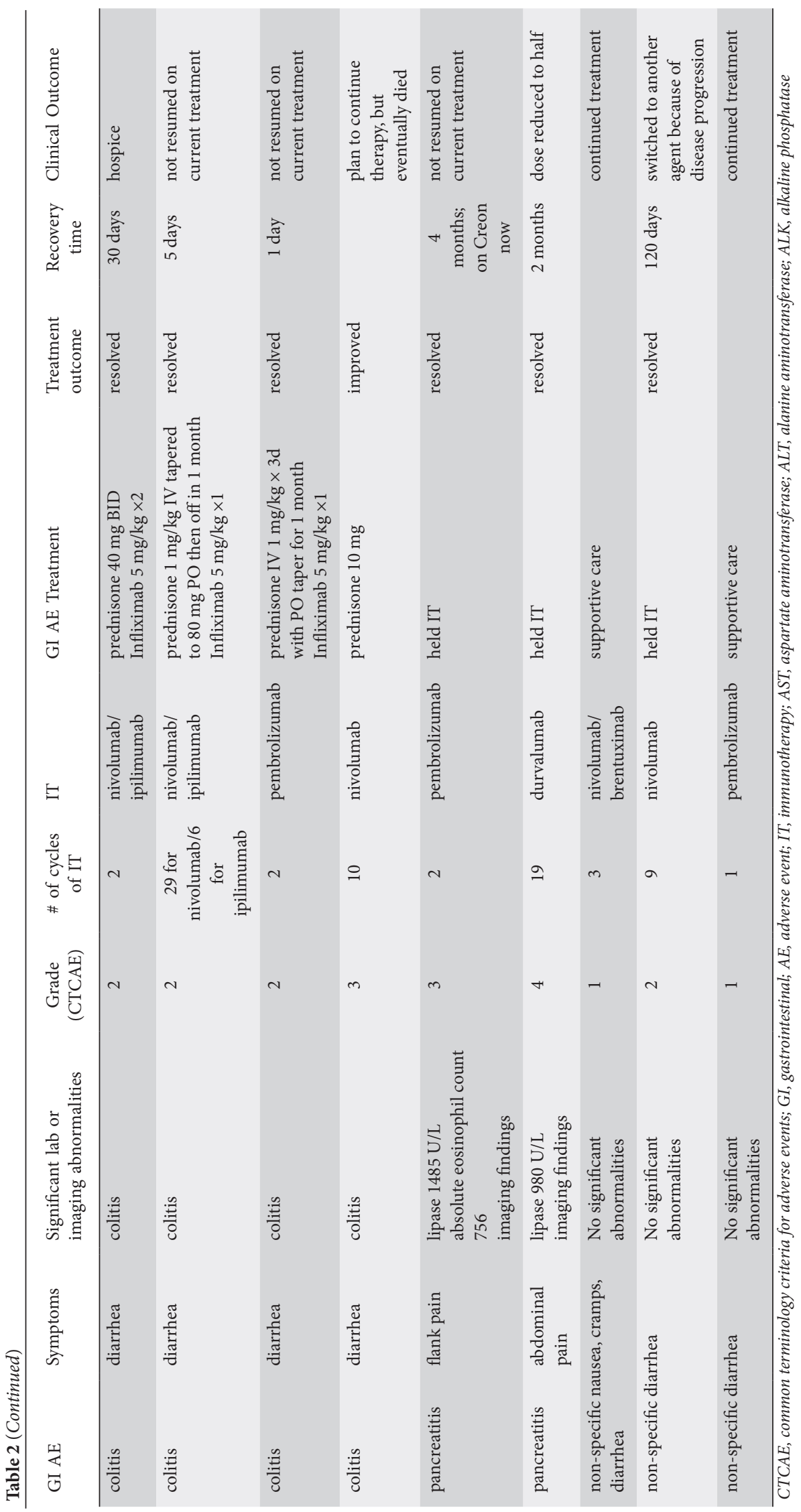




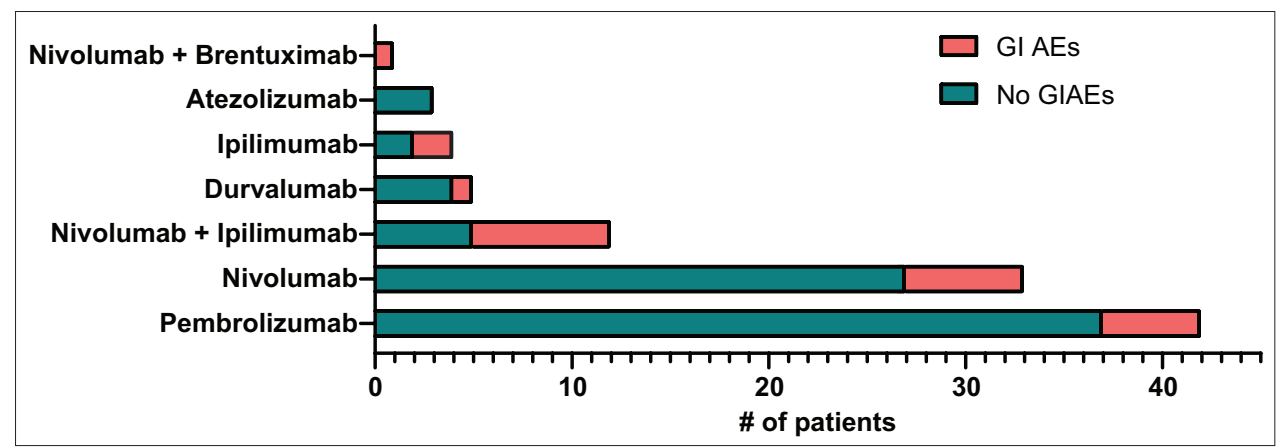

Figure 2 Distribution of side effects for each chemotherapy regimen. This graph depicts the relative number of adverse events related to the gastrointestinal tract for each immunotherapy regimen. Notably, the majority of patients on combo therapy with nivolumab + ipilimumab had GI AEs GI, gastrointestinal; $A E$, adverse event

compared to those who did not receive IFX. The use of IFX in treating irAEs is suggested as second line after patients do not respond to steroids [14]. Most patients do respond well to IFX [14]. Given the quick recovery rate of patients on IFX-on the order of 10 days in our study - this therapy may be used in an attempt to avoid the prolonged use of steroids, which leads to higher infection rates [15]. Although IFX offers an alternative to prolonged steroid use, its use also comes with risks. Patients being considered for IFX must have up-to-date screening for hepatitis B as well as tuberculosis [16]. In addition, patients with severe heart failure or concurrent elevated serum aminotransferases may not be ideal candidates for IFX [16].

While most patients had resolution of their GI AEs, only 7/22 were maintained on their original treatment regimen, in some cases at a lower dosage. This statistic is marred by the fact that some patients expired prior to engaging in conversation about future treatment. Notably, no patients with colitis were able to continue on their current regimen, because of the physician's clinical judgement, intolerance to further sessions, or death. In general, severe colitis occurs in $<10 \%$ of the cases and ICIs may be restarted up until grade 3 colitis if the benefits outweigh the risks $[5,16,17]$. The more conservative approach in our cohort may be in line with the documented fatalities associated directly with GI AEs. A meta-analysis that represented the largest evaluation of fatal toxic effects of ICIs reported colitis to be the most frequent cause of anti-CTLA- 4 toxicity death, while antiPD1 was more often associated with hepatitis [18].

Our cohort of patients experiencing GI AE on immunotherapy had a high mortality rate at $41 \%$, despite having high ECOG performance status between 0 and 1 , but no deaths were directly recorded to be attributed to the GI AE event, although one patient was hospitalized for GI AE and discharged directly home to hospice. In general, fatal toxicities directly attributed to GI AE are rare [15], but many of our patients expired after discontinuing their immunotherapy and were in the process of evaluating other treatment options. Given the high mortality rate of patients with GI AE findings in our cohort, it is important to screen and treat GI AEs early on, with the hope of either continuing on the current treatment regimen or planning ahead for secondary options.

Our study is limited by the number of cases of ICI GI AEs, as well as the lack of complete data recorded in the EHR. Future work will be directed towards expanding the cohort so that more data can be collected regarding other ICI regimens of which we only had a few cases (i.e., atezolizumab, durvalumab). Overall, our study depicts colitis and transaminitis to be the most prevalent GI AEs encountered in patients on immunotherapy. Notably, the combination of nivolumab with ipilimumab was highly associated with colitis. Cases of GI AEs do resolve with proper treatment and care, and IFX is a particularly effective agent for quickly improving symptomatology in colitis. Despite resolution, most patients are unable to continue on the current treatment regimen and still have a high mortality rate, though deaths are not directly attributable to GI AEs.

\section{Summary Box}

\section{What is already known:}

- Gastrointestinal toxicities are a common sideeffect of immunotherapy, with colitis being charted as the most common side-effect

- Steroids and sometimes anti-inflammatory immunomodulators are used to treat gastrointestinal adverse events that are high grade on the Common Terminology Criteria for Adverse Events scale

- Fatalities resulting directly from these gastrointestinal adverse events are uncommon

\section{What the new findings are:}

- Colitis is a particularly common side-effect in patients on dual therapy with nivolumab/ipilimumab

- The majority of patients are not maintained on their current immunotherapy regimen, leading to a search for alternatives

- While most cases of gastrointestinal adverse events due to cancer immunotherapy achieve resolution, the overall end mortality rate of patients experiencing these events is still quite high 


\section{References}

1. Azoury SC, Straughan DM, Shukla V. Immune checkpoint inhibitors for cancer therapy: clinical efficacy and safety. Curr Cancer Drug Targets 2015;15:452-462.

2. Postow MA, Sidlow R, Hellmann MD. Immune-related adverse events associated with immune checkpoint blockade. $N$ Engl J Med 2018;378:158-168

3. Pento, JT. Monoclonal antibodies for the treatment of cancer. Anticancer Res 2017;37:5935-5939.

4. Yoest, JM. Clinical features, predictive correlates, and pathophysiology of immune-related adverse events in immune checkpoint inhibitor treatments in cancer: a short review. Immunotargets Ther 2017;6:73-82.

5. Som A, Mandaliya R, Alsaadi D, et al. Immune checkpoint inhibitor-induced colitis: a comprehensive review. World J Clin Cases 2019;7:405-418.

6. Bajwa R, Cheema A, Khan T, et al. Adverse effects of immune checkpoint inhibitors (programmed death-1 inhibitors and cytotoxic T-lymphocyte-associated protein-4 inhibitors): results of a retrospective study. J Clin Med Res 2019;11:225-236.

7. Rajha E, Chaftari P, Kamal M, et al. Gastrointestinal adverse events associated with immune checkpoint inhibitor therapy. Gastroenterol Rep (Oxf). 2020;8:25-30.

8. Liu YH, Zang XY, Wang JC, et al. Diagnosis and management of immune related adverse events (irAEs) in cancer immunotherapy. Biomed Pharmacother 2019;120:109437.

9. Paydas, S. Gastrointestinal side effects of immune checkpoint inhibitors: new topic for gastroncologists. Hematol Med Oncol 2019;4:1-9.
10. Baxi S, Yang A, Gennarelli RL, et al. Immune-related adverse events for anti-PD-1 and anti-PD-L1 drugs: Systematic review and meta-analysis. BMJ 2018;360:k793.

11. Hodi FS, O'Day SJ, McDermott DF, et al. Improved survival with ipilimumab in patients with metastatic melanoma. $N$ Engl J Med 2010;363:711-723.

12. Wang PF, Chen Y, Song SY, et al. Immune-related adverse events associated with Anti-PD-1/PD-L1 treatment for malignancies: a meta-analysis. Front Pharmacol 2017;18:730.

13. Soldatos TG, Dimitrakopoulou-Strauss A, Larribere L, et al. Retrospective side effect profiling of metastatic melanoma combination therapy ipilimumab-nivolumab using adverse event data. Diagnostics 2018;8:E76.

14. Johnston RL, Lutzky J, Chodhry A, et al. Cytotoxic T-lymphocyteassociated antigen 4 antibody-induced colitis and its management with infliximab. Dig Dis Sci 2008;54:2538.

15. Wang DY, Salem JE, Cohen JV, et al. Fatal toxic effects associated with immune checkpoint inhibitors: A systematic review and meta-analysis. JAMA Oncol 2018;4:1721-1728.

16. Bristol-Myers Squibb Company. Opdivo immune-mediated adverse reactions management guide. Available from: https://www. opdivohcp.com/assets/commercial/us/opdivo-hcp-pan-tumor/ en/pdf/Immune_Mediated_Adverse_Management_Guide.pdf [Accessed 7 September 2020].

17. Prieux-Klotz C, Dior M, Damotte D, et al. Immune checkpoint inhibitor-induced colitis: diagnosis and management. Target Oncol 2017; 12:301-308.

18. Jiang $\mathrm{Y}$, Zhang N, Pang H, et al. Risk and incidence of fatal adverse events associated with immune checkpoint inhibitors: a systematic review and meta-analysis. Ther Clin Risk Manaj 2019;15:293-302. 